\title{
Atención Dental Durante la Pandemia COVID-19
}

\author{
Dental Care During COVID-19 Pandemic
}

\author{
Daniela Catalina Martínez Camus \& Sebastián Rodolfo Yévenes Huaiquinao
}

\begin{abstract}
MARTÍNEZ, C. D. C. \& YÉVENES, H. S. R. Atención dental durante la pandemia COVID-19. Int. J. Odontostomat., 14(3):288295, 2020.

RESUMEN: COVID-19 es una enfermedad infecciosa producida por el virus SARS-CoV-2. Entre la población de alto riesgo de contagio se encuentra el personal de salud. Particularmente, la atención dental por sus características es una situación de riesgo para los profesionales odontólogos y los auxiliares dentales. Por lo tanto, es necesario caracterizar las principales vías de transmisión del virus SARS-CoV-2 y describir las medidas para prevenir el contagio en el contexto de la atención dental. La bibliografía de la bases de datos Pubmed y Scielo, además, de guías clínicas sobre atención odontológica, indican que la práctica dental es una situación de alto riesgo debido a que los procedimientos dentales producen gotas y aerosoles, potencialmente contaminados. Además, existe contacto estrecho entre el clínico y el paciente. Se recomienda que los odontólogos suspendan la atención electiva, y solo realicen procedimientos de urgencia o emergencia. En casos de atención dental de urgencia, la higiene de manos, tanto del paciente como del clínico, es indispensable. Se deben utilizar barreras de protección personal, disminuir los procedimientos que generen aerosoles o gotas, utilizar aislación con goma dique y eyección mediante una técnica a 4 manos. Además, es necesaria una adecuada desinfección del instrumental y las superficies clínicas antes y después de la atención. A la fecha, no existe un protocolo universal para la atención durante el brote COVID-19, las recomendaciones están en constante evolución según se determinan nuevas características del virus SARS-CoV-2. Sin embargo, se deben tomar medidas de precaución antes, durante y después de la atención dental para reducir el riesgo de contagio entre el paciente y el personal de salud dental.
\end{abstract}

PALABRAS CLAVE: COVID-19, SARS-CoV-2, dental, odontólogo.

\section{INTRODUCCION}

En diciembre del 2019, en la ciudad de Wuhan se reportaron casos de individuos que presentaban una neumonía de causa desconocida, que un grupo de investigadores atribuyó a un coronavirus nuevo (Zhu et al., 2020). En febrero del 2020, el virus fue nombrado como el coronavirus 2 del síndrome respiratorio agudo grave o SARS-CoV-2 (Gorbalenya et al., 2020). La enfermedad se extendió rápidamente a otros países (Liu et al., 2020), y en marzo 2020 la Organización Mundial de la Salud (OMS) declaró que el brote de la enfermedad por el SARS-CoV-2 era una pandemia (World Health Organization, 2020a).

COVID-19 es la enfermedad infecciosa causada por el virus SARS-CoV-2, y provoca un cuadro clínico variable. Se han detectado pacientes asintomáticos, y casos sintomáticos en que los individuos experimentan tos seca, fiebre, dificultad para respirar, fatiga y dolor muscular. En los casos más gra- ves se reporta una neumonía severa en que el paciente requiere hospitalización, y asistencia respiratoria mediante ventilación mecánica. La enfermedad puede comprometer la vida del paciente (Chen et al., 2020b; Huang et al., 2020; Wang et al., 2020; Meng et al., 2020). Hasta la fecha 12 de abril del 2020, según el reporte $\mathrm{N}^{\circ} 83$ de la OMS sobre la situación COVID19 , se han confirmado 1.696 .588 casos y 105.952 personas han muerto por esa enfermedad. A la misma fecha, los países de América latina con mayor número de casos confirmados son Brasil, Ecuador y Chile (World Health Organization, 2020b).

Aunque es más probable un cuadro clínico severo en individuos con comorbilidades, las personas de todas las edades son susceptibles a la enfermedad COVID-19 (Chen et al., 2020b; Shi et al., 2020; Guan et al., 2020a). Los individuos que están en contacto estrecho con pacientes positivos tienen mayor 
riesgo de infección, como los trabajadores de la salud (Peng et al., 2020; Wold Health Organization 2020c; Alharbi et al., 2020; Zimmermann \& Nkenke, 2020). Particularmente, la atención dental requiere del contacto estrecho entre el odontólogo y el paciente. Además, los procedimientos generan una gran cantidad de gotas y aerosoles potencialmente contaminados. Por lo tanto, la atención dental puede ser de alto riesgo (Peng et al.; Alharbi et al.,; Meng et al.; Ge et al., 2020; Zimmermann \& Nkenke), y provocar la infección cruzada entre los pacientes y los odontólogos.

La presente revisión tiene como objetivo caracterizar las principales vías de transmisión del virus SARS-CoV-2 en la atención dental y describir las principales medidas para prevenir el contagio durante los procedimientos dentales.

\section{MATERIAL Y MÉTODO}

Se realizó una revisión de la literatura a partir de un total de 34 referencias bibliográficas. Se utilizaron artículos nacionales e internacionales de las bases de datos Pubmed, Scielo y Elsevier. Los términos de búsqueda utilizados fueron SARS-CoV-2, COVID19, dental, transmisión, odontología. Además, se analizaron guías clínicas sobre la atención de pacientes en el contexto COVID-19 publicadas por la OMS u otras instituciones de salud. La última búsqueda se realizó el 13 de abril del 2020. Se seleccionaron los artículos que tenían como máximo 5 años o menos de haberse publicado. Posteriormente se analizó la calidad y validez de los artículos selecciona-dos para realizar una adecuada revisión.

\section{RESULTADOS Y DISCUSIÓN}

Vías de transmisión del SARS-CoV-2. Se ha descrito que la transmisión del SARS-CoV-2 ocurre principalmente entre personas a través de gotas respiratoria ( $\mathrm{Ge}$ et al.). Estas partículas se emiten al toser, estornudar o hablar, y pueden contener saliva u otros fluidos que contengan el virus (Peng et al.; Alharbi et al.,; Meng et al.; van Doremalen et al., 2020; Zimmermann \& Nkenke). Las gotas grandes caen al suelo rápidamente; por lo tanto, la transmisión por gotas requiere de una proximidad física, permitiendo que las partículas emitidas por un individuo infectado sean inhaladas por un individuo susceptible (Ge et al.).
Es necesario mantener una distancia de 1.5 metros para evitar el contagio (Trilla, 2020). Sin embargo, también se ha reportado que el SARS-CoV-2 podría ser transportado por el aire a través de aerosoles, producidos durante ciertos procedimientos. En tal caso las gotitas evaporadas tienen una velocidad de sedimentación baja, por lo que pueden permanecer en el aire por un tiempo prolongado, antes de ingresar al tracto respiratorio o contaminar las superficies (Ge et al.; van Doremalen et al.). Se ha planteado que el contacto manual con los fómites o superficies contaminadas, puede provocar el ingreso del virus a través de las membranas mucosas orales, nasales y oculares, al tocar esas mucosas con las manos contaminadas (Peng et al.; Alharbi et al.; Meng et al.; Ge et al.). Se ha establecido que el virus puede permanecer viable en superficies durante al menos tres horas, e incluso días dependiendo del material, la humedad y la temperatura (van Doremalen et al.).

Los pacientes sintomáticos producen la mayor cantidad de contagios. Sin embargo, los pacientes asintomáticos e incluso los individuos en periodo de incubación de la enfermedad son portadores del SARS-CoV-2, y pueden contagiar (Bai et al., 2020; Chan et al., 2020; Rothe et al., 2020; To et al., 2020; Meng et al.). El período de incubación del virus se ha estimado en 6 días en promedio, aunque algunos estudios sugieren que puede ser de 14 días (Backer et al., 2020; Li et al., 2020; Guan et al., 2020b). En tales casos es difícil identificar a los pacientes y ponerlos en cuarentena, lo que provoca una complicación para el control de la enfermedad (Trilla). Además, aún no se determina si los pacientes en fase de recuperación son una fuente de transmisión (Meng et al.). La transmisión vertical (de las madres a sus recién nacidos) aún no está descartada (Chen et al., 2020a). Adicionalmente, se ha reportado que puede encontrarse ARN del SARS-CoV-2 en heces de un paciente. Sin embargo, la ruta de transmisión fecal-oral aún debe investigarse (Peng et al.).

Riesgo de transmisión en la atención dental. La necesidad de tratamiento dental urgente disminuyó en un $38 \%$ durante el brote COVID-19 (Guo et al., 2020). Lo anterior indica que aún durante el estado de pandemia, sigue existiendo necesidad de atención dental. Sin embargo, la atención dental es una situación de alto riesgo, en que los pacientes, profesionales odontólogos y auxiliares dentales pueden estar expuestos a microorganismos patógenos, incluidos virus que infectan la cavidad oral y el tracto respiratorio (Peng et al.; Alharbi et al.; Meng et al.). 
Los procedimientos dentales implican un contacto estrecho con los pacientes, en que no se puede respetar la recomendación de distanciamiento social. Además, existe constante exposición a fluidos corporales, como la saliva, que contiene el virus SARS-CoV2 (Peng et al.; To et al.; Meng et al.; Zimmermann \& Nkenke). La transmisión de gotitas y aerosoles contaminadas con SARS-CoV-2 son la preocupación más importante en la atención dental, debido a que durante los tratamientos dentales de rutina se genera gran cantidad de estas partículas, mezcladas con la saliva y sangre del paciente (Peng et al.; Alharbi et al.,). Particularmente, los dispositivos dentales como la pieza de mano de alta velocidad, equipos de ultrasonido y la jeringa triple, funcionan a alta velocidad, con aire y agua, generando una gran cantidad de gotas y aerosoles (Peng et al.; Alharbi et al.,; Meng et al.). Además, los dentistas manejan instrumental cortopunzante (Peng et al.; Meng et al.). También se ha descrito que las células epiteliales del tejido lingual muestran una alta expresión de la enzima convertidora de angiotensina II (ACE2), principal receptor del SARSCoV-2 en las células humanas (Peng et al.; Xu et al., 2020). Por lo que la mucosa de la cavidad oral puede ser una ruta de ingreso (Xu et al.). Por otro lado, el virus puede persistir en superficies (van Doremalen et al.), por lo que podría estar presentes en el instrumental odontológico o en las superficies ambientales de la clínica dental (Peng et al.).

\section{Medidas para el control de la infección COVID-19 previo a la atención dental}

1. Evaluación de los integrantes del equipo de salud dental. La Asociación Dental Americana (ADA) recomienda que el dentista y su equipo hayan recibido vacunación para la influenza, y que si presentan algún síntoma sospechoso de COVID-19 no asistan a trabajar (American Dental Association 2020a). Se debe determinar que integrantes del equipo tienen un mayor riesgo de contraer COVID-19, ya sea porque son de la tercera edad, tengan una afección preexistente u otra razón. Además, los integrantes del equipo deber estar alertas a cualquier síntoma respiratorio y verificar su temperatura dos veces al día (American Dental Association, 2020a).

2. Necesidad de atención dental. Es recomendable una consulta por teléfono o videollamada, antes de asistir al centro dental. Esto permite evitar asistencias innecesarias (American Dental Association, 2020a; Subsecretaría de Salud Pública, 2020; Meng et al.; Zimmermann \& Nkenke). Además, permite determi- nar la necesidad de atención dental, ya que se deben posponer todas las atenciones que no sean de urgencia o emergencia ( American Dental Association, 2020a; Subsecretaría de Salud Pública; Alharbi et al.,; Meng et al.; Yang et al., 2020; Ge et al.).

3. Evaluación del paciente. En caso de que un paciente deba asistir a la clínica dental, se recomienda que los profesionales identifiquen casos sospechosos de COVID-19 previo a la atención, realizando una evaluación minuciosa de cada paciente (Peng et al.; Alharbi et al.,; Meng et al.; Ge et al.; Zimmermann \& Nkenke). Antes de la atención, se debe registrar la temperatura corporal, idealmente mediante un termómetro de frente (Meng et al.). Luego, se debe aplicar un cuestionario que permitan identificar el estado de salud, y posibles síntomas de infección. Además, es necesario preguntar sobre historial de viajes y el contacto con personas infectadas (Peng et al.; Subsecretaría de Salud Pública; Meng et al.; Ge et al.).

4.Recomendaciones en la sala de espera. Los pacientes deben asistir con mascarilla y mantener distanciamiento social. La cantidad de pacientes en la sala de espera debe reducirse al mínimo, para eso es necesario espaciar las citas de atención (American Dental Association, 2020a; Subsecretaría de Salud Pública), y evitar que los pacientes traigan acompañantes, excepto en los casos en que requieran asistencia (American Dental Association, 2020a). Además, los tiempos de espera y contacto deben ser cortos (American Dental Association, 2020a; Subsecretaría de Salud Pública). Es recomendable retirar todos los elementos no necesarios de la sala de espera (ej.; revistas) que puedan ser tocados, y que sean difícil de desinfectar. La sala de espera debe estar sala ventilada y con acceso a higiene de manos. Los pacientes deben realizar un lavado de manos con agua y jabón o con desinfectantes a base de alcohol, antes y después de la atención dental (American Dental Association, 2020a; Subsecretaría de Salud Pública).

\section{Categorización del paciente que asiste a la con- sulta dental}

La evaluación previa a la atención dental permite categorizar a los pacientes según riesgo de contagio de COVID-19. Además, se debe evaluar la necesidad y urgencia del tratamiento.

1. Pacientes no sospechosos. Se puede realizar la atención dental de individuos no sospechosos y que no supongan un riesgo (pacientes sin fiebre $u$ otros 
síntomas consistentes con la infección COVID-19), utilizando medidas de protección, y solo en caso de urgencias (American Dental Association, 2020a; Peng et al.; Subsecretaría de Salud Pública; Alharbi et al.,; Meng et al.). En aquellos pacientes que tienen fiebre, pero esta se encuentra asociada al diagnóstico dental (por ejemplo, fiebre e inflamación intraoral), y no hay otros signos/síntomas de infección COVID-19, se puede realizar la atención utilizando barreras de protección (American Dental Association, 2020b). Peng et al. indican que en tal caso se deben reducir los procedimientos que generen aerosoles. Por otro lado, Alharbi et al., indican que se pueden realizar procedimientos que generan aerosoles, solo cuando constituyan una urgencia, como el tratamiento del dolor dental severo por inflamación pulpar, fractura de un diente vital y trauma dental con avulsión/luxación, (Alharbi et al.,). Además, en casos impostergables se puede realizar ajustes de prótesis en pacientes oncológicos, reparación de prótesis fija fracturada que cause lesiones en los tejidos blandos y tratamiento de enfermedad periodontal aguda (Alharbi et al.,). Es recomendable utilizar un box dental para los pacientes no sospechosos distinto al de pacientes sospechosos (Subsecretaría de Salud Pública).

2. Pacientes sospechosos. Los tratamientos en paciente sospechosos deben posponerse, si es posible. Si no lo es, idealmente el paciente debe ser derivado para su atención en un servicio de salud en que se puedan aplicar medidas apropiadas para disminuir la transmisión (American Dental Association, 2020 b). Se recomienda que los procedimientos se realicen en una sala de aislamiento contra infecciones transmitidas por el aire (AIIR) o en salas de presión negativa, que reduzcan la diseminación del virus (American Dental Association, 2020b; Alharbi et al.,; Zimmermann \& Nkenke).

Durante la atención de urgencia de individuos sospechosos se deberá evitar la generación de aerosol (Alharbi et al.,). Alharbi et al., indican que los tratamientos de urgencia incluyen el dolor dental severo por inflamación pulpar, dolor dental severo de un diente vital fracturado, trauma dental con avulsión/luxación, alveolitis post-extracción, pericoronaritis, fracturas maxilofaciales, absceso dental/periodontal localizado y fractura de aparatos de ortodoncia que causen laceración de tejidos blandos, pero estos procedimientos deben manejarse sin generar aerosoles. Adicionalmente, se debe educar a los individuos sospechosos asintomáticos sobre los signos de la infección COVID-19 e indicarles acudir a un centro de salud en caso de presentarlos, además de cuarentena por 14 días (Peng et al.). Los autores también recomiendan que los pacientes sospechosos que presenten síntomas sean derivados a un centro de salud (Peng et al.; Meng et al.).

3. Pacientes confirmados. La atención de pacientes confirmados con COVID-19 debe posponerse (American Dental Association, 2020a; Peng et al.; Subsecretaría de Salud Pública; Alharbi et al.,; Meng et al.). Si la atención es impostergable, debe ser coordinada con el médico, y realizarse en una sala AIIR o en salas de presión negativa con un mínimo de 12 cambios de aire por hora o al menos $160 \mathrm{~L} / \mathrm{s}$ por paciente (American Dental Association, 2020a), e idealmente en un entorno hospitalario (Peng et al.; Alharbi et al.,; Zimmermann \& Nkenke). Se recomiendan realizar solo tratamientos de emergencia, como la atención de fracturas maxilofaciales inestables que pueden comprometer las vías respiratorias del paciente, procesos infecciosos en tejidos blandos con inflamación intraoral o extraoral que puede comprometer las vías respiratorias del paciente, y sangrado postoperatorio que no puede ser controlado (Alharbi et al.,; Zimmermann \& Nkenke).

4. Pacientes recuperados. No existe un consenso de cuándo un paciente que fue diagnosticado con la enfermedad puede interrumpir el aislamiento en el hogar. Algunos autores han indicado que el paciente debe ser considerado portador del virus hasta después de 30 días de confirmar su recuperación con una prueba de laboratorio (Alharbi et al.,). Otros indican que se debe esperar un tiempo de 7 días sin presentar sintomatología (American Dental Association, 2020a). Es necesario el juicio clínico para determinar la urgencia de la atención, y postergarla de ser posible.

\section{Medidas para el control de la infección COVID-19 durante la atención dental}

1. Lavado de manos. El lavado de manos es una medida crítica para reducir el riesgo de transmisión de microorganismos entre el clínico y el paciente (American Dental Association, 2020a; Peng et al.; Subsecretaría de Salud Pública; Ge et al.; World Health Organization, 2020c; Zimmermann \& Nkenke). Cuando las manos están visiblemente sucias, la higiene debe realizarse con agua y jabón durante 40 a 60 segundos, con una técnica apropiada. Si están visiblemente limpias, se puede usar un desinfectan- 
te a base de alcohol durante $20-30$ segundos (American Dental Association, 2020a; Subsecretaría de Salud Pública 2020; Meng et al. 2020; World Health Organization, 2020d). Si el jabón o alcohol no se encuentran disponibles, el agua clorada $(0,05 \%)$ es una opción, aunque es frecuente que provoque dermatitis (World Health Organization, 2020d). El lavado debe realizarse antes de colocarse los elementos de protección personal y después de retirarlos, antes y después del contacto con el paciente o sus secreciones (sangre, saliva u otros fluidos), antes y después de un procedimiento dental, después del contacto con el entorno y el equipamiento dental (Peng et al.; Subsecretaría de Salud Pública; Ge et al.). Además, los profesionales dentales deben evitar tocarse sus propios ojos, boca y nariz (Peng et al.; World Health Organization, 2020d).

\section{Desinfección del instrumental y superficies am-} bientales. Como el SARS-CoV-2 puede persistir en las superficies, es necesaria una adecuada desinfección de todo el instrumental y equipamiento dental, y de todas las superficies ambientales antes de la atención y entre pacientes (Meng et al.; Zimmermann \& Nkenke). El instrumental debe ser desinfectado o esterilizado, según corresponda (Peng et al.). Para la desinfección algunos estudios recomiendan etanol al $62-71 \%$, peróxido de hidrógeno al $0,5 \%$ o hipoclorito de sodio al 0,1\% durante 1 minuto (Ge et al.; World Health Organization, 2020d). La OMS recomienda usar alcohol etílico al $70 \%$ para desinfectar instrumentos entre usos (por ejemplo, termómetros); e hipoclorito de sodio al 0,5\% para desinfectar superficies (Kampf et al., 2020). Estudios indican que otros agentes, como el cloruro de benzalconio al $0,05-0,2 \%$ o el digluconato de clorhexidina al 0,02\% son menos efectivos (Kampf et al.). Mantener un ambiente limpio y seco en el consultorio dental también ayudaría a disminuir la persistencia del SARS-CoV-2 (Peng et al.; Ge et al.).

3. Barreras de protección estándar. Se recomienda que todo el equipo de salud, incluido el profesional odontólogo y el auxiliar dental, utilicen barreras de protección personal. Se debe incluir el uso de máscaras faciales y gafas protectoras, para proteger los ojos. Estos elementos deben ser utilizados en todo momento, y desinfectarse entre pacientes. Es necesario el uso de gorro y guantes desechables, bata impermeable, ropa quirúrgica y cobertor de calzado desechables, para protegerse de secreciones potencialmente infectadas (American Dental Association, 2020a; Peng et al.; Subsecretaría de Salud Pública; Meng et al.; Zimmermann \& Nkenke). Los trajes clínicos y zapatos deben ser distintos a la vestimenta de circulación fuera de los establecimientos de salud (Subsecretaría de Salud Pública). Además, es necesario utilizar una máscara filtrante. Se recomienda el uso de máscaras $\mathrm{N}-95$ o máscaras FFP2, para la atención de pacientes durante el examen clínico o durante procedimientos que no generen aerosoles (Peng et al.; Subsecretaría de Salud Pública; Meng et al.; Zimmermann \& Nkenke). Para procedimientos generadores de aerosoles o cirugías realizadas en pacientes con COVID-19, se deben utilizar mascaras N-99 o FFP3 (Peng et al.; Meng et al.; Ge et al.; Zimmermann \& Nkenke).

4. Enjuague oral. Los estudios describen que se debe indicar al paciente que realice un enjuague bucal antimicrobiano previo a la atención dental con el objetivo de reducir la carga microbiana en la saliva (Peng et al.; Subsecretaría de Salud Pública; Alharbi et al.,; Ge et al.; Zimmermann \& Nkenke). Dado que SARSCoV-2 es vulnerable a la oxidación, se recomienda el enjuague bucal con peróxido de hidrógeno al $1 \%$ o povidona al $0,2 \%$ por al menos 15 segundos (Peng et al.; Alharbi et al.,; Zimmermann \& Nkenke), otros autores indican que debe ser por 30 segundos (American Dental Association, 2020a; Subsecretaría de Salud Pública).

\section{Recomendaciones sobre procedimientos. Se de-} ben minimizar los procedimientos que generen gotas o aerosoles, como el uso de la jeringa triple, la pieza de mano de alta velocidad o instrumentos de ultrasonido (American Dental Association, 2020a; Peng et al.; Subsecretaría de Salud Pública; Alharbi et al.,; Meng et al.). En caso de utilizar piezas de mano de alta velocidad, estas deben contar con válvulas anti-retorno, para reducir significativamente el flujo de micro-organismos al interior de la pieza de mano y la unidad dental (American Dental Association, 2020a; Peng et al.). Además, se recomienda que los procedimientos generadores de aerosoles se realicen en la última atención del día (American Dental Association, 2020a). Es preferible utilizar dispositivos manuales para la eliminación de caries y alisado periodontal, a fin de minimizar la generación de aerosol (Meng et al.; Peng et al.; Alharbi et al., American Dental Association, 2020a).

Todos los estudios recomiendan el uso de goma dique y eyector de saliva para minimizar la salpicadura provocada en los procedimientos dentales. Además, la atención dental a 4 manos es necesaria para lograr una adecuada aislación y eyección (American Dental Association, 2020a; Peng et al.; Subsecretaría de Salud Pública; Alharbi et al.,; Meng et al.). 
Siempre que sea posible deben usarse instrumentos y dispositivos desechables y de un solo uso, para reducir los riesgos de infección cruzada (Alharbi et al).

En caso de necesitar una radiografía, se deben preferir las radiografías extraorales, como la radiografía panorámica, o la tomografía computarizada de haz cónico. Lo anterior se debe a que las radiografías intraorales estimulan la salivación, la tos y el reflejo nauseoso (American Dental Association, 2020a; Subsecretaría de Salud Pública; Alharbi et al.,; Meng et al.).

En caso de procedimientos quirúrgicos en que se deba suturar, se debe preferir la sutura reabsorbible (American Dental Association, 2020a; Subsecretaría de Salud Pública; Alharbi et al.,; Meng et al.). En caso de heridas, se recomienda enjuagarla lentamente y usar el eyector para evitar salpicaduras (Zimmermann \& Nkenke).

Menge et al., recomiendan que cuando existan traumas o lesiones maxilofaciales que provoquen el ingreso inmediato de los pacientes al hospital, de ser posible se debe tomar una tomografía de tórax, para descartar la infección pulmonar. Esto debido a que la prueba de reacción en cadena de la polimerasa (PCR) puede tomar mucho tiempo (Meng et al.).

6. Uso de fármacos. Hasta la fecha, no existe evidencia de que el uso del ibuprofeno u otros antinflamatorios no esteroidales (AINEs) pueda provocar un agravamiento de la condición de salud en pacientes con COVID-19. A pesar de eso, Alharbi et al., indican que se debe evitar su uso cuando se requiera tratamiento farmacológico para disminuir el dolor de origen dental, en casos sospechosos y confirmados de COVID-19. Por otro lado, la ADA recomienda el uso de los AINEs en combinación con acetaminofeno para el manejo del dolor dental en pacientes adultos inmunocompetentes (American Dental Association, 2020a).

Medidas de control de la infección COVID-19 después de la atención dental

1. Desinfección de instrumental y superficies ambientales. Al finalizar la jornada, también se deben realizar los procedimientos de limpieza y desinfección de instrumental y equipamiento odontológico, y de las superficies y entornos (World Health Organization 2020c).
2. Seguimiento de los pacientes. Se debe solicitar un numero de contacto a los pacientes y acompañantes, para informar si otro paciente que asistió en la misma fecha fue confirmado posteriormente con infección por SARS-CoV-2 (Meng et al.).

\section{CONCLUSIÓN}

No existe un protocolo universal para atención dental en casos sospechosos o confirmados de COVID-19, lo que puede provocar un aumento de transmisión del virus en la atención dental. La literatura muestra que es recomendable que los profesionales suspendan la atención electiva, y solo realicen procedimientos de urgencia o emergencia que no puedan ser postergados. Por lo tanto, el criterio profesional es necesario para determinar la necesidad de atención y el riesgo del paciente. En casos de atención de urgencia, se describe que la higiene de manos, tanto del paciente como del clínico, es indispensable. Se recomienda el uso de barreras de protección personal, la disminución de los procedimientos que generen aerosoles o gotas, la aislación con goma dique y la eyección mediante una técnica a 4 manos. Además, se recomienda realizar una adecuada desinfección del instrumental y las superficies clínicas. Todo paciente debe ser considerado potencialmente contaminante $y$, por lo tanto, se deben adoptar medidas de protección antes, durante y después de la atención dental. La información de las características de la infección por SARS-CoV-2 está en constante evolución, por lo tanto, es necesario que los protocolos de atención dental durante el brote COVID-19 sean constantemente actualizados, con el objetivo de disminuir el riesgo de contagio.

MARTíNEZ, C. D. C. \& YÉVENES, H. S. R. Dental care during COVID-19 pandemic. Int. J. Odontostomat., 14(3):288295, 2020.

ABSTRACT: COVID-19 is an infectious disease caused by the SARS-CoV-2 virus. Among the population at high risk of contagion is health workers. In particular, dental care due to its characteristics is a risk situation for dental professionals and dental assistants. Therefore, it is necessary to characterize the main routes of transmission of the SARSCoV-2 virus and describe the measures to prevent contagion in the context of dental care. The bibliography of the Pubmed and Scielo databases, in addition to clinical guidelines on dental care, indicate that dental practice is a high-risk situation because dental procedures produce potentially contaminated 
drops and sprays. Furthermore, there is close contact between the clinician and patient. It is recommended that dentists discontinue elective care, and only perform urgent or emergency procedures. In cases of emergency dental care, hand hygiene, both for the patient and the clinician, is essential. Personal protection barriers should be used, procedures that generate aerosols or drops should be reduced, isolation with rubber dam and ejection using a 4handed technique should be used. In addition, adequate disinfection of instruments and clinical surfaces is required before and after care. To date, there is no universal protocol for care during the COVID-19 outbreak, the recommendations are constantly evolving as new characteristics of the SARSCoV-2 virus are determined. However, precautionary measures should be taken before, during, and after dental care to reduce the risk of contagion between the patient and dental health personnel.

KEY WORDS:COVID-19, SARS-CoV-2, dental, dentist.

\section{REFERENCIAS BIBLIOGRÁFICAS}

Alharbi, A.; Alharbi, S. \& Alqaidi, S. Guidelines for dental care provision during the COVID-19 pandemic. Saudi Dent. J., 2020. DOI: https://doi.org/10.1016/j.sdentj.2020.04.001

American Dental Association (ADA). ADA Interim Guidance for Minimizing Risk of COVID-19 Transmission. American Dental Association, 2020a. Disponible en: https://www.ada.org/ $\sim$ / $\mathrm{m}$ e d i a / C P S / F i l e s / C O V I D / ADA_COVID_Int_Guidance_Treat_Pts.pdf

American Dental Association. ADA Interim Guidance for Management of Emergency and Urgent Dental Care. American Dental Association, 2020b. Disponible en: https:// www. ada.org/ / media/CPS/Files/COVID/

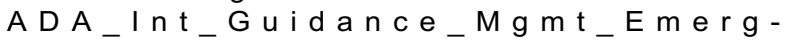
Urg_Dēntal_CōVID19.pdf

Backer, J. A.; Klinkenberg, D. \& Wallinga, J. Incubation period of 2019 novel coronavirus (2019-nCoV) infections among travellers from Wuhan, China, 20-28 January 2020. Euro Surveill., 25(5), 2020. DOI: https://doi.org/10.2807/15607917.ES.2020.25.5.2000062

Bai, Y.; Yao, L.; Wei, T.; Tian, F.; Jin, D. Y.; Chen, L. \& Wang, M. Presumed asymptomatic carrier transmission of COVID-19. JAMA, 2020. DOI: https://doi.org/10.1001/jama.2020.2565

Chan, J. F. W.; Yuan, S.; Kok, K. H.; To, K. K. W.; Chu, H.; Yang, J.; Poon, R. W. S.; Tsoi, H. W.; Lo, S. K. F.; Chan, K. H.; et al. A familial cluster of pneumonia associated with the 2019 novel coronavirus indicating person-to-person transmission: a study of a family cluster. Lancet, 395(10223):514-23, 2020.

Chen, H.; Guo, J.; Wang, C.; Luo, F.; Yu, X.; Zhang, W.; Li, J.; Zhao, D.; Xu, D.; Gong, Q.; et al. Clinical characteristics and intrauterine vertical transmission potential of COVID-19 infection in nine pregnant women: a retrospective review of medical records. Lancet, 395(10226):809-15, 2020a.

Chen, N.; Zhou, M.; Dong, X.; Qu, J.; Gong, F.; Han, Y.; Qu, Y.; Wang, J.; Liu, Y.; Wei, Y.; et al. Epidemiological and clinical characteristics of 99 cases of 2019 novel coronavirus pneumonia in Wuhan, China: a descriptive study. Lancet, 395(10223):507-13, 2020b.
Ge, Z. Y.; Yang, L. M.; Xia, J. J.; Fu, X. H. \& Zhang, Y. Z. Possible aerosol transmission of COVID-19 and special precautions in dentistry. J. Zhejiang Univ. Sci. B, 2020. DOI: https://doi.org/ 10.1631/jzus.B2010010

Gorbalenya, A. E.; Baker, S. C.; Baric, R. S.; de Groot, R. J.; Drosten, C.; Gulyaeva, A. A.; Haagmans, B. L.; Lauber, C.; Leontovich, A. M.; Neuman, B. W.; et al. Severe acute respiratory syndrome-related coronavirus: The species and its viruses - a statement of the Coronavirus Study Group. bioRxiv, 2020. DOI: https://doi.org/10.1101/2020.02.07.937862

Guan, W. J.; Liang, W. H.; Zhao, Y.; Liang, H. R.; Chen, Z. S.; Li, Y. M.; Liu, X. Q.; Chen, R. C.; Tang, C. L.; Wang, T.; et al. Comorbidity and its impact on 1590 patients with Covid-19 in China: A Nationwide Analysis. Eur. Respir. J., 2000547, 2020a. DOI: https://doi.org/10.1183/13993003.00547-2020

Guan, W. J.; Ni, Z. Y.; Hu, Y.; Liang, W. H.; Ou, C. Q.; He, J. X.; Liu, L.; Shan, H.; Lei, C. L.; Hui, D. S. C.; et al. Clinical characteristics of 2019 novel coronavirus infection in China. N. Engl. J. Med., 2020b. DOI: https://doi.org/10.1056/ NEJMoa2002032

Guo, H.; Zhou, Y.; Liu, X. \& Tan, J. The impact of the COVID-19 epidemic on the utilization of emergency dental services. $J$. Dent. Sci., 2020. DOI: https://doi.org/10.1016/ j.jds.2020.02.002

Huang, C.; Wang, Y.; Li, X.; Ren, L.; Zhao, J.; Hu, Y.; Zhang, L.; Fan, G.; Xu, J.; Gu, X.; et al. Clinical features of patients infected with 2019 novel coronavirus in Wuhan, China. Lancet, 395(10223):497-506. 2020.

Kampf, G.; Todt, D.; Pfaender, S. \& Steinmann, E. Persistence of coronaviruses on inanimate surfaces and their inactivation with biocidal agents. J. Hosp. Infect., 104(3):246-51, 2020.

Li, Q.; Guan, X.; Wu, P.; Wang, X.; Zhou, L.; Tong, Y.; Ren, R.; Leung, K. S. M.; Lau, E. H. Y.; Wong, J. Y.; et al. Early transmission dynamics in Wuhan, China, of novel coronavirus-infected pneumonia. N. Eng. J. Med., 382(13):1199-207, 2020.

Liu, T.; Hu, J.; Xiao, J.; He, G.; Zang, M.; Rong, Z.; Lin, L.; Zhong, H.; Huang, Q.; Deng, A.; et al. Time-varying transmission dynamics of Novel Coronavirus Pneumonia in China. bioRxiv, 2020. DOI: https://doi.org/10.1101/2020.01.25.919787

Meng, L.; Hua, F. \& Bian, Z. Coronavirus disease 2019 (COVID-19): emerging and future challenges for dental and oral medicine. J. Dent. Res., 2020. DOI: https://doi.org/10.1177/ 0022034520914246

Peng, X.; Xu, X.; Li, Y.; Cheng, L.; Zhou, X. \& Ren, B. Transmission routes of 2019-nCoV and controls in dental practice. Int. J. Oral Sci., 12:9, 2020.

Rothe, C.; Schunk, M.; Sothmann, P.; Bretzel, G.; Froeschl, G.; Wallrauch, C.; Zimmer, T.; Thiel, V.; Janke, C.; Guggemos, W.; et al. Transmission of 2019-nCoV infection from an asymptomatic contact in Germany. N. Engl. J. Med., 382(10):970-1, 2020.

Shi, Y.; Yu, X.; Zhao, H.; Wang, H.; Zhao, R. \& Sheng, J. Host susceptibility to severe COVID-19 and establishment of a host risk score: findings of 487 cases outside Wuhan. Crit. Care, 24(1):108, 2020.

Subsecretaría de Salud Pública. Orientaciones para Atención Odontológica en Fase IV COVID-19. Santiago de Chile, Subsecretaría de Salud Pública, División de Prevención y Control de Enfermedades, Departamento Salud Bucal, Ministerio de Salud, Gobierno de Chile, 2020. Disponible en: https:/ /diprece.minsal.cl/wp-content/uploads/2020/03/ORIENTACIONES-ATENCION-ODONTOLOGICAS-COVID-19-.pdf

To, K. K.; Tsang, O. T.; Chik-Yan Yip, C.; Chan, K. H.; Wu, T. C.; Chan, J. M. C.; Leung, W. S.; Chik, T. S.; Choi, C. Y.; Kandamby, D. H.; et al. Consistent detection of 2019 novel coronavirus in saliva. Clin. Infect. Dis., ciaa149, 2020. DOI: https://doi.org/ 10.1093/cid/ciaa149 
Trilla, A. One world, one health: The novel coronavirus COVID-19 epidemic. Med. Clin. (Barc.), 154(5):175-7, 2020.

van Doremalen, N.; Bushmaker, T.; Morris, D. H.; Holbrook, M. G.; Gamble, A.; Williamson, B. N.; Tamin, A.; Harcourt, J. L.; Thornburg, N. J.; Gerber, S. I.; et al. Aerosol and surface stability of SARS-CoV-2 as compared with SARS-CoV-1. N. Engl. J. Med., 382(16):1564-7, 2020

Wang, D.; Hu, B.; Hu, C.; Zhu, F.; Liu, X.; Zhang, J.; Wang, B.; Xiang, H.; Cheng, Z.; Xiong, Y.; et al. Clinical characteristics of 138 hospitalized patients with 2019 novel coronavirus-infected pneumonia in Wuhan, China. JAMA, 323(11):1061-9, 2020.

World Health Organization (WHO). Virtual Press Conference on COVID-19. Ginebra, World Health Organization, 2020a. Disponible en: https://www.who.int/docs/default-source/ coronaviruse/transcripts/who-audio-emergencies-coronaviruspress - conference-full-a nd-fin al11 mar2020.pdf?sfvrsn=cb432bb3_2

World Health Organization (WHO). Coronavirus Disease 2019 (COVID-19) Situation Report - 83. Ginebra, World Health Organization, 2020b. Disponible en: https://www.who.int/docs/ default-source/coronaviruse/situation-reports/20200412-sitrep83-covid-19.pdf?sfvrsn=697ce98d_4

World Health Organization (WHO). Risk assessment and management of exposure of health care workers in the context of COVID-19. Ginebra, World Health Organization, 2020c. Disponible en: https://apps.who.int/iris/bitstream/handle/10665/ 331496/WHO-2019-nCov-HCW_risk_assessment-2020.2eng.pdf?sequence=1\&isAllowed $=\mathrm{y}$

World Health Organization (WHO). Water, Sanitation, Hygiene And Waste Management for COVID-19. Ginebra, World Health Organization, 2020d. Disponible en: https://www.who.int/ publications-detail/water-sanitation-hygiene-and-wastemanagement-for-covid-19

Xu, H.; Zhong, L.; Deng, J.; Peng, J.; Dan, H.; Zeng, X.; Li, T. \& Chen, Q. High expression of ACE2 receptor of 2019-nCoV on the epithelial cells of oral mucosa. Int. J. Oral Sci., 12:8, 2020.

Yang, Y; Zhou, Y; Liu, X; Tan, J. Health services provision of 48 public tertiary dental hospitals during the COVID-19 epidemic in China. Clin. Oral Investig., 2020. DOI: https://doi.org/10.1007/ s00784-020-03267-8

Zhu, N.; Zhang, D.; Wang, W.; Li, X.; Yang, B.; Song, J.; Zhao, X.; Huang, B; Shi, W.; Lu, R.; et al. A novel coronavirus from patients with pneumonia in China, 2019. N. Engl. J. Med., 382(8):727-33, 2020.

Zimmermann, M. \& Nkenke, E. Approaches to the management of patients in oral and maxillofacial surgery during COVID-19 pandemic. J. CranioMaxillofac. Surg., 2020. DOI: https://doi.org/ 10.1016/j.jcms.2020.03.011

\author{
Dirección para correspondencia: \\ Facultad de Odontología \\ Universidad de Chile \\ Santiago \\ CHILE
}

Email: daniela.martinez.c@ug.uchile.cl sebastian.yevenes@ug.uchile.cl

Recibido: 14-04-2020

Aceptado:15-04-2020 